\title{
Study the Characteristic of Planer and Sandwich PSi Gas Sensor (Comparative Study)
}

\author{
Alwan M. Alwan ${ }^{1}$ (D) Ruaa A Abbas ${ }^{2}$. Amer B. Dheyab ${ }^{2}$ \\ Received: 6 December 2016 / Accepted: 28 February 2018 / Published online: 27 March 2018 \\ (C) The Author(s) 2018
}

\begin{abstract}
In this work, two configurations (planner and sandwich) structures of (Al/n PSi/n-Si/Al) porous silicon (PSi) gas sensor have been fabricated and tested extensively for $\mathrm{CO}_{2}$ gas molecules. Two laser wavelength infrared and violet of $(810 \mathrm{~nm})$ and $(405 \mathrm{~nm})$ was used in the laser assisting etching process on the n-type silicon substrate. The gas detection characteristic of planner and sandwich configuration were studied under different condition. The resistance measurement, for the planner and $\mathrm{J}-\mathrm{V}$ characteristic for sandwich structures, which was analyzed based on the nano-sized silicon, porosity, layer thickness, and the effective dielectric constant of the PSi layer. The SEM image of the PSi layer showed the formation pore-like structure with cylindrical and rectangular pore shape with different dimensions for infrared illuminated PSi and crossed pores-like structure with randomly distributed for violet illuminated PSi. The sensing mechanism for sandwich structure configuration is governed by the porous silicon parameters, while for planer configuration the silicon channel among the pore has a significant role in sensing process.
\end{abstract}

Keywords Porous silicon · Surface morphology $\cdot$ Gas sensor $\cdot$ Carbon dioxide

\section{Introduction}

There is a growing importance in developing able and economically gas sensors for detecting the leakage of different gases harmful. Environment air quality has improved considerably in the last few decades due to modern air pollution legislation. Nevertheless, there is strong evidence suggesting that exposure to air pollution, even at the levels commonly achieved nowadays in developed countries, leads to adverse health effects. A great variety of different sensors prepared of the PSi has been made-up over recent years. Has been detection many of organic materials, including DNA, measured by PSi sensors have been reported. Aroutiounian [1-3]. PSi can be considered as a

Amer B. Dheyab

amer.aljoburi@yahoo.com

1 School of Applied sciences, University of Technology, Baghdad, Iraq

2 Ministry of Science and Technology, Baghdad, Iraq silicon crystal having a network of voids in it. The nanosized voids in the bulk silicon result in the different form of the structure of pores and channels surrounded by a skeleton of crystalline $\mathrm{Si}$ nano wires [4]. Laser assisted etching (LAE) was the mechanism used in the formation of PSi layer on the Si surface in hydrofluoric acid with employing photonic source [5]. Due to light absorption process, the size and the density of the nanocrystallites and PSi layer thickness can be modulated. The density of $\mathrm{Si}$ nanocrystallites is decreased with increasing the etching laser wavelength, while the porous layer thickness is increased with increase the etching laser wavelength [6]. The requirement of industry and personal circumstances led to the development of efficient gas detection configuration. The gas sensing model sensitivity, response and recovery times depended on the PSi gas sensor configuration [7]. Carbon dioxide detection is currently in demand in the major fields such as indoor air quality monitoring $[8,9]$. There are several groups in the development of an alternative sensor for $\mathrm{CO} 2$ monitoring, which is cheap and efficient [10]. The ability of the PSi to sense at room temperature organic vapors such as acetone, trichloroethylene, and hexane, which are harmful to human health. The electrical 
current (DC) and photoluminescence at the presence of an organic vapor have been done to examine the sensor performance with the organic vapors. The result showed was that the PSi layer has high sensitivity to the organic vapors. PSi is very effective material to enhance the optical properties of Si [11].

In this work, a comparative study was carried out on two fabricated porous silicon gas sensor configuration (planner and sandwich). The electrical behavior each sensor is discussed based on the mechanism of the current flow within the porous silicon matrix and the porous silicon parameters.

\section{Fabrication and Experimental Details}

The porous silicon gas sensor was fabricated using silicon

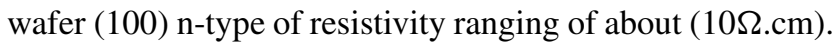
The silicon wafer is cutting into $(2.4 \times 2.4 \mathrm{~cm})$ in dimension. The etching process was carried out in Teflon cell where the silicon substrate acts as an anode while the cathode is a platinum ring with $15 \mathrm{~min}$ etching time and $(25 \mathrm{~mA} / \mathrm{cm} 2)$ current density. The etching solution consists of the mixture (HF: Ethanol: H2O 1:1:1). Two types of porous samples were prepared using laser assisted etching (LAE), sample A with illumination wavelength infrared of $(810 \mathrm{~nm})$ and illumination intensity of $\left(2 \mathrm{~W} / \mathrm{cm}^{2}\right)$ and sample B with the violet illumination wavelength of $(405 \mathrm{~nm})$ and illumination intensity $\left(2 \mathrm{~mW} / \mathrm{cm}^{2}\right)$. After the formation of the PSi layer, the required deposition Aluminum electrode is deposited onto the surface of the porous silicon and the rare surface of crystalline silicon to produce electrical contacts for device testing. Vacuum thermal evaporation process was used to make the electrodes. The Very thin layer is a deposit on the upper PSi surface with thickness (10-20 nm), to make the sandwich structure of (Al/nona-PSi/n-Si/Al) as shown in the Fig. 1a. The planer configuration was obtained deposit two different E-letter Aluminum electrode on the PSi layer. The current-voltage characteristic was performed in the small sealed dark chamber under a controlled gas flow at various temperatures $25-150 \mathrm{C}^{\circ}$; calibrated thermocouple monitored the temperature. A DC power supply and 8846AFluke6$1 / 2$ Digit precision multimeter, while the resistance measurement was carried out with (Hank 365 Multimeter).

In the same way, to obtain a homogeneous distribution of the gas molecule on the porous silicon gas sensor, the measurements were performed after 1 minute of turning on the gas flow. The measured concentration of $\mathrm{CO}_{2}$ gas is about $5 \mathrm{ppm}$. The surface morphology of the porous silicon was studied using (AIS3200C) scanning electron microscopy SEM. The PL spectra were obtained at room temperature using He-Cd laser (320 nm, $10 \mathrm{mw}$, for excitation. Fourier transfer infrared (FTIR) spectrometer analyses were carried out by (Per Kin -Elmer spectrometer).

\section{Result and Discussion}

\subsection{Characteristic of Porous Silicon Layer}

A gravimetric technique was used to determine the porosity and layer thickness of the porous layer. Porous sample A and $\mathrm{B}$ the porosity is $(68 \%)$ and $(73 \%)$ respectively, while the layer thickness is (23.56) and (15) respectively. The porous layer thickness of sample $A$ is much greater than that of sample B this is due to the increase of the absorption depth with decreasing the illumination wavelength. The absorption depth is about $(12.9 \mu \mathrm{m})$ for $(810 \mathrm{~nm})$ while for $(405 \mathrm{~nm})$ the depth of about $(100 \mathrm{~nm})$. So the etching process due to the photon absorption and the required e-h pair's generation for silicon dissolution process will occur at a region away from the porous layer and inside the deep silicon regions.

Figure 2 illustrates the surface morphology of sample A and $\mathrm{B}$ respectively with two different surface morphologies. For sample A the porous layer looks like pore-like structure with different pore size and pore shape, the pore shape is spherical and rectangular with a large density of the formation pores. The statistical distribution of pores varies
Fig. 1 PSi sample (a) planer (b) sandwich
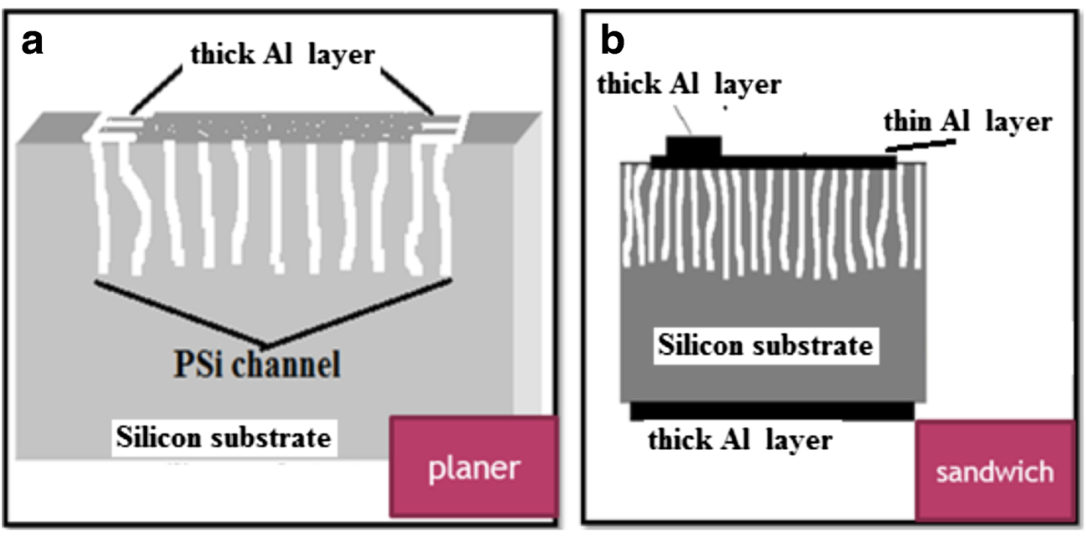


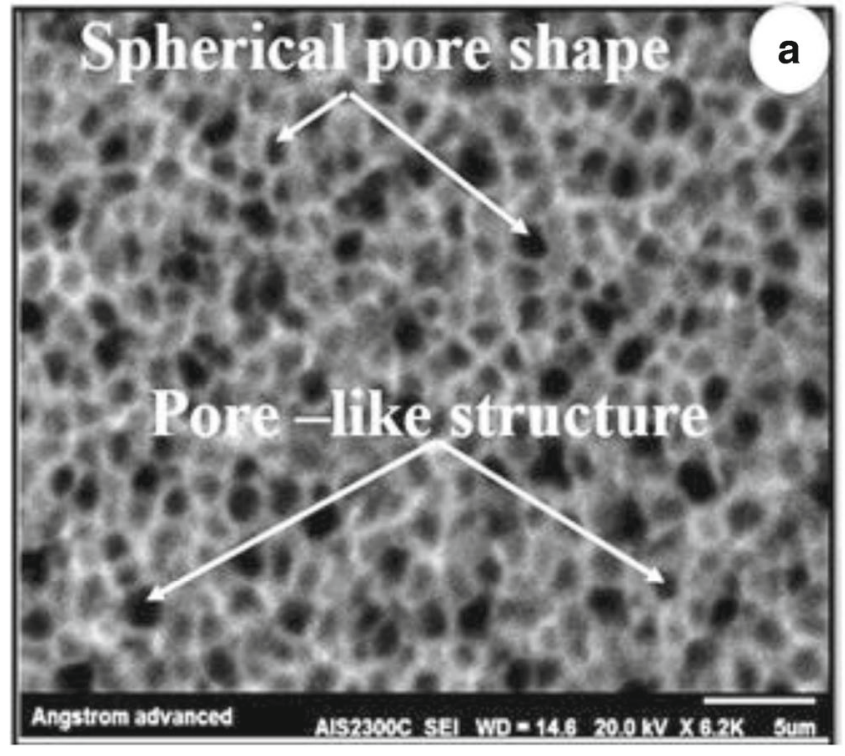

Fig. 2 SEM image (top-view) of (a) sample A (b) sample B

from $(0.1$ to $4 \mu \mathrm{m})$ and the peak of the distribution located at $(1 \mu \mathrm{m})$. For sample B.

The morphology of the porous looks like layer. A cross pores-like structure with different trench sizes. According to the analysis of the SEM image the morphology of the

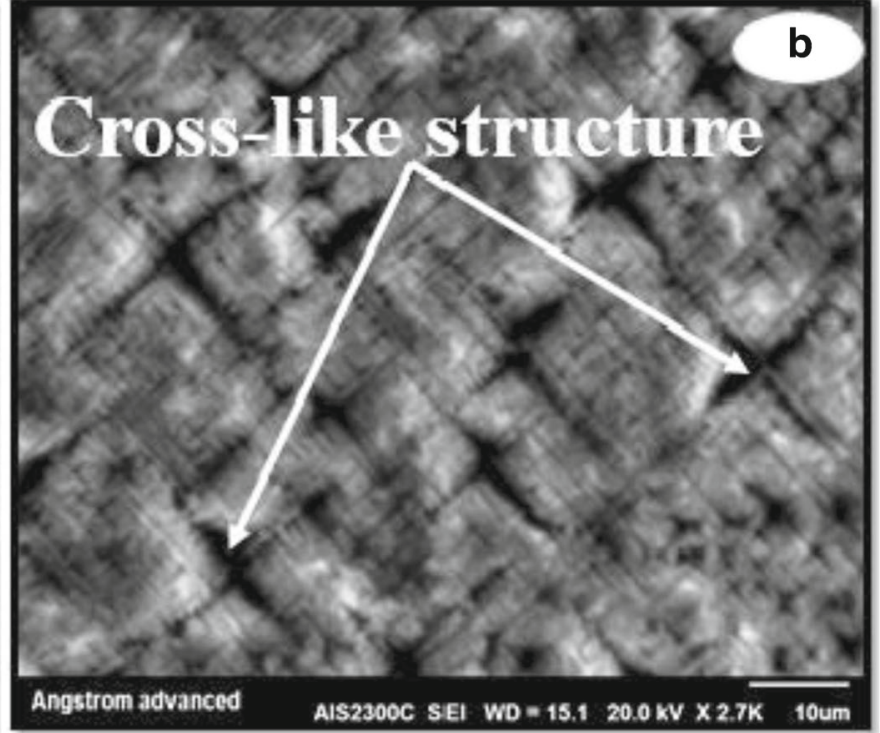

porous formed under violet illumination present side branch that propagates perpendicularly to their main direction of the growth, leading to form a cross-like structure. Cláudia studied the formation of this infrequent morphology, according to the analysis of [12], the removing of the silicon
Fig. 3 FTIR absorption spectra of (a) sample A (b) sample B
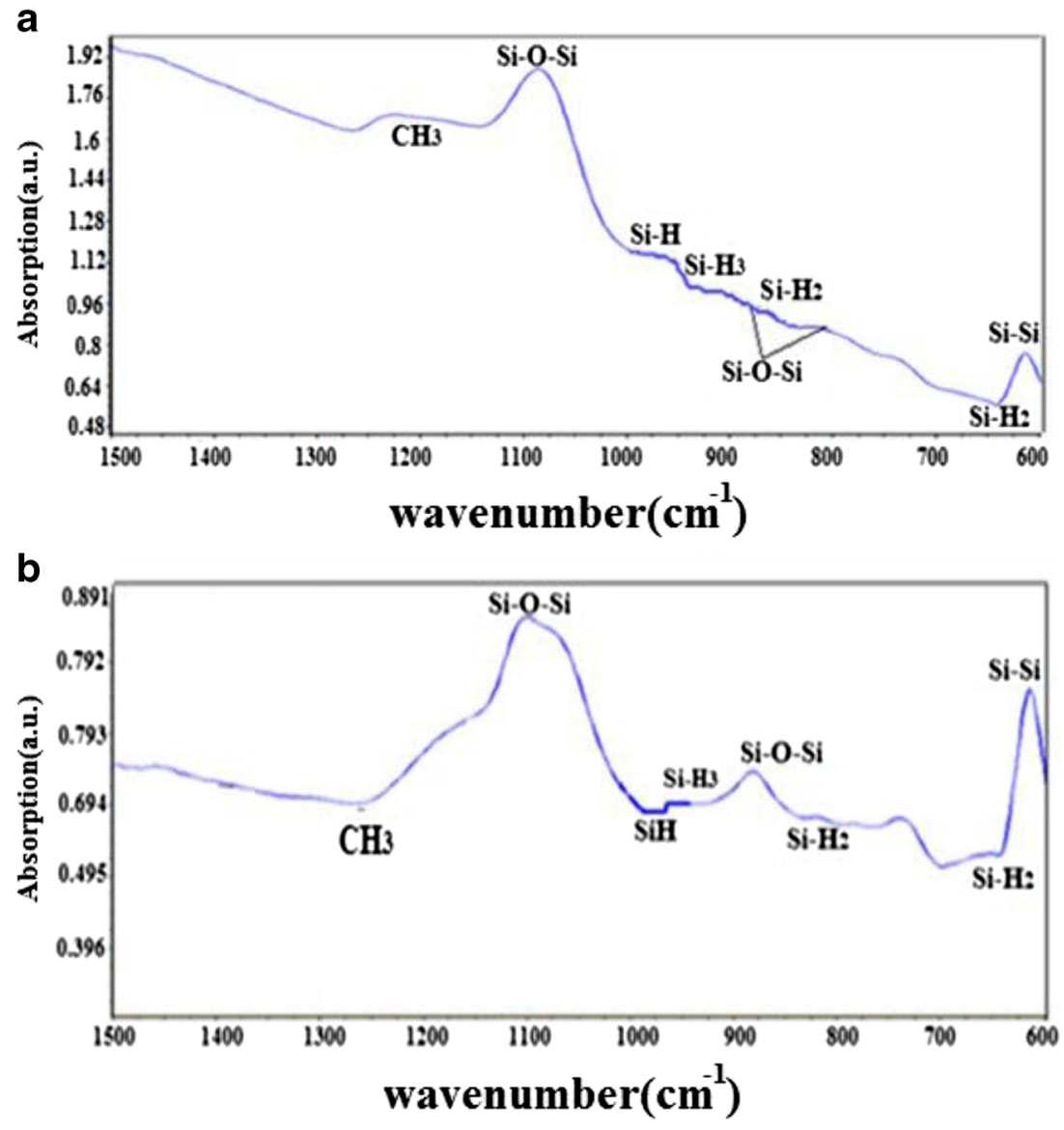


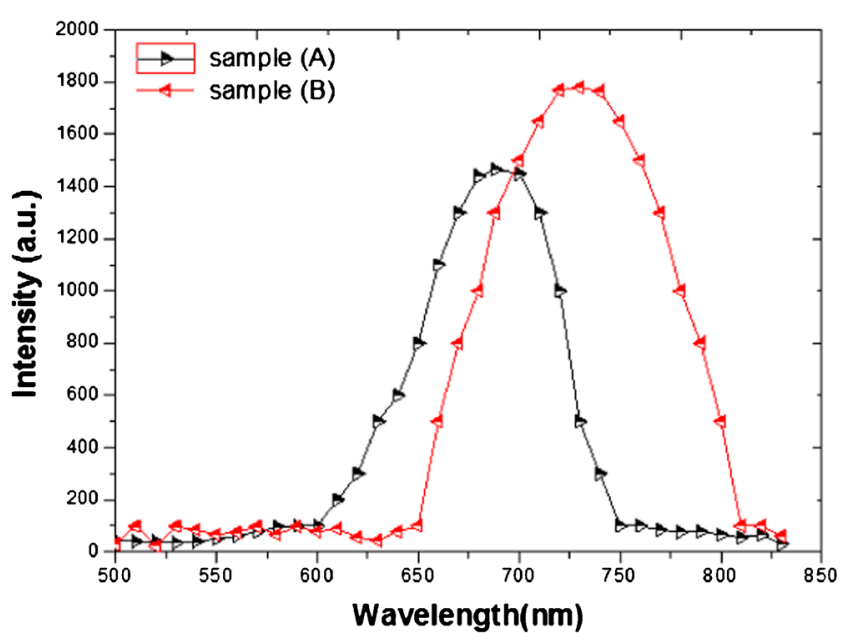

Fig. 4 illustrates the PL spectra of PSi

atoms will enhance the surface inhomogeneity due to the local changes in the electric field distribution. The absorption of the violet wavelength $405 \mathrm{~nm}$ will occur within the $\mathrm{Si}$ surface and under very shallow region below the $\mathrm{Si}$ surface, where the absorption depth of $405 \mathrm{~nm}$ is about $(100 \mathrm{~nm})$. This will restrict the etching in the surface and the photogenerated of e-h pairs will affect the electric field distribution leading to improve, the in homogeneous of the pore growth.

The chemical species on porous silicon surfaces is determined by FTIR signals from porous silicon. Hydrogen covers the dangling bonds of silicon after the etching process, so silane $\mathrm{SiH}_{\mathrm{x}}(\mathrm{x}=1,2,3)$ groups and $\mathrm{Si}-\mathrm{O}-\mathrm{Si}$ termination are formed on the surface of the prepared porous silicon. Surface morphology governs types of such group and the intensity of the absorption [13]. Figure $3 \mathrm{a}$ and $\mathrm{b}$ shows the FTIR spectra of IR and violet illumination that presents that the absorption intensity of sample A due to dihydrides $(\mathrm{Si}-\mathrm{H} 2)$, trihydrides $(\mathrm{Si}-\mathrm{H})$, and $\mathrm{Si}-\mathrm{O}-\mathrm{Si}$ is higher than that of sample B.The existence of Si-O-Si termination in both porous silicon samples is due to the oxidation process which occurs due to the presence of $\mathrm{H} 2 \mathrm{O}$ molecule in the etching solution so the PSi layer is partially oxidized layer.

The (PL) spectra of porous silicon for both samples are displaced in Fig. 4 shown it can recognize that the PL spectrum of sample B, the PL intensity is higher than that of sample A and the PL peak intensity is located at $(688 \mathrm{~nm})$ and $(630 \mathrm{~nm})$ respectively. The increasing of the illumination wavelength (decreasing the photon energy) leads to synthesize porous layer with larger silicon nano size as shown on in Eq. (1) [14]. The dependence of the PL spectra on the silicon nano size was correlated with porous silicon morphologies.
The energy of the porous layer, $\mathrm{E}_{\mathrm{g}(\mathrm{PSi})}$ varies according to the silicon nano size $(\mathrm{L})$.

$E g_{(\mathrm{PSi})}=E g_{(\mathrm{S})}+88.34 / L^{1.37}$

The penetration is increased in the etching rate with laser power density which is due to an increase in the number of generated (e-h) pairs and subsequently leads to the faster etching process. The number of photogenerated (e-h) pairs is given by:

$G_{e-h}=\alpha I / h \nu$

Where $(\alpha)$ is the absorption coefficient, $(I)$ is the laser intensity and $(h v)$ is the photon energy.

\subsection{Performance of Porous Silicon Gas Sensors}

The gas sensing performance of two types of porous silicon gas sensors configurations (planer and sandwich structure) for different kinds of surface morphologies were studied at different operation temperatures.

\subsubsection{Sandwich Structure Mode}

The gas sensing mechanism of this mode of operation is based on the response of the current-voltage characteristic across the porous silicon /crystalline silicon junction. All the obtained result show rectifying behavior rather than a linear relationship.

Figure 5 shows the $\mathrm{J}-\mathrm{V}$ characteristics of sample $\mathrm{A}$ and $\mathrm{B}$ illuminated porous silicon respectively in the sandwich $(\mathrm{Al} / \mathrm{nPSi} / \mathrm{nSi} / \mathrm{Al})$ structure configuration mode at different temperatures in the absence of $\mathrm{CO}_{2}$ gas molecules. All measurement were done under forwarding bias in the range from $(0-5 v)$.

From these curves, we can observe the dependence of the current density on the characteristic of the porous silicon layer, where the current density is increased with increasing the porosity and with decreasing the layer thickness of the porous layer and, the following facts can be concluded.

1. For both sample the forward current density increase slightly with increasing the operation temperatures from $25 \mathrm{C}^{\circ}$ to $150 \mathrm{C}^{\circ}$.

2. The current passing through the violet illuminated sample B, in general, higher than that of infrared illuminated porous sample A.

The equation gives the dependence of the current density on the porous silicon parameters Eq. (3) [15]. The thickness and porosity both types of illumination.

$\mathbf{J}_{\mathbf{P S i}}=\epsilon_{\mathrm{rps}} \epsilon_{\mathbf{0}} \mu_{\mathbf{e f f}} \mathbf{V}^{\mathbf{2}} / \mathbf{d}^{3}$

Where $\epsilon_{\mathbf{r P S i}}$ is a dielectric constant of PSi, $\epsilon_{\mathbf{O}}$ dielectric constant of air, $\mu_{\mathbf{e f f}}$ is the mobility of the charge carriers 

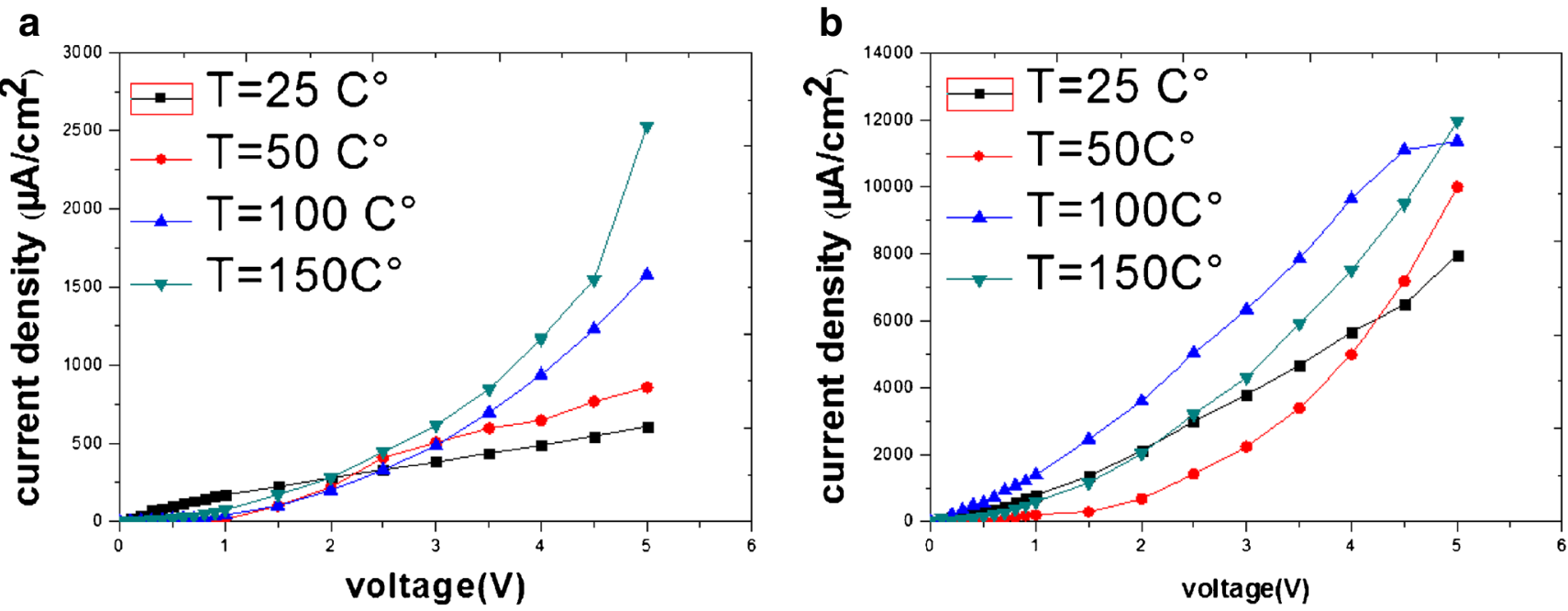

Fig. 5 The J-V characteristic of porous silicon sandwich structure (Al/nPSi/n-Si/Al)with (a) sample A (b) sample B

in the PSi layer, $\mathrm{V}$ the applied voltage, and $\mathrm{d}$ the layer thickness of PSi. The current density strongly affected by the layer thickness and hence depended on the porosity of porous layer. According to the Eq. (3).

Figure 6 presents the $\mathrm{J}-\mathrm{V}$ characteristics of PSi sandwich structure with the presence of fixed $\mathrm{CO}_{2}$ concentration of about $(5 \%) \mathrm{ppm} \mathrm{CO} 2$. From this number, it is clear to that the $\mathrm{J}-\mathrm{V}$ characteristic is still rectifying. The values of the current density passing through both samples with the presence of $\mathrm{CO}_{2}$ gas are higher than that of the case without gas, so this variation in the current density indicates that the PSi sensor was very sensitive to $\mathrm{CO}_{2}$ gas molecules. The current variation in the sandwich structure of sample B is higher than that of sample $\mathrm{A}$, and this variation is related to desorption of the $\mathrm{CO}_{2}$ molecule on the porous silicon layer due to the Vander Waals interaction. The $\mathrm{CO}_{2}$ desorption will lead to modifying the dielectric constant of the porous layer. The dependence of the dielectric $\epsilon \mathrm{r}$ Psi on the porosity of the porous layer and the embedding medium $\left(\epsilon_{\mathrm{rpore}}\right)$ is given by the Eq. (4) [16].

$\in \mathrm{r}_{\mathrm{PSi}}=(1-\mathrm{P} \%) \epsilon_{\mathrm{rSi}}^{1 / 3}+\mathrm{P} \% \epsilon_{\mathrm{r} \text { pore }}^{1 / 3}$

Where $\mathrm{P} \%$ porosity of the porous layer, $\epsilon \mathrm{r}$ pore dielectric constant of the embedding medium $\left(\mathrm{CO}_{2}\right.$ molecule). The value of the dielectric constant of the porous layer in the presence of $\mathrm{CO}_{2}$ gas molecules filling the pores is about (3.86267233) and about (4.71059865) for sample A and sample B respectively.

The values of the dielectric constant for $\mathrm{CO}_{2}$ molecule is about (1.000921) without gas condensation effect, and (1.6) with gas condensation effect. The condensation process for the gas and vapor molecule in the porous structure occurred
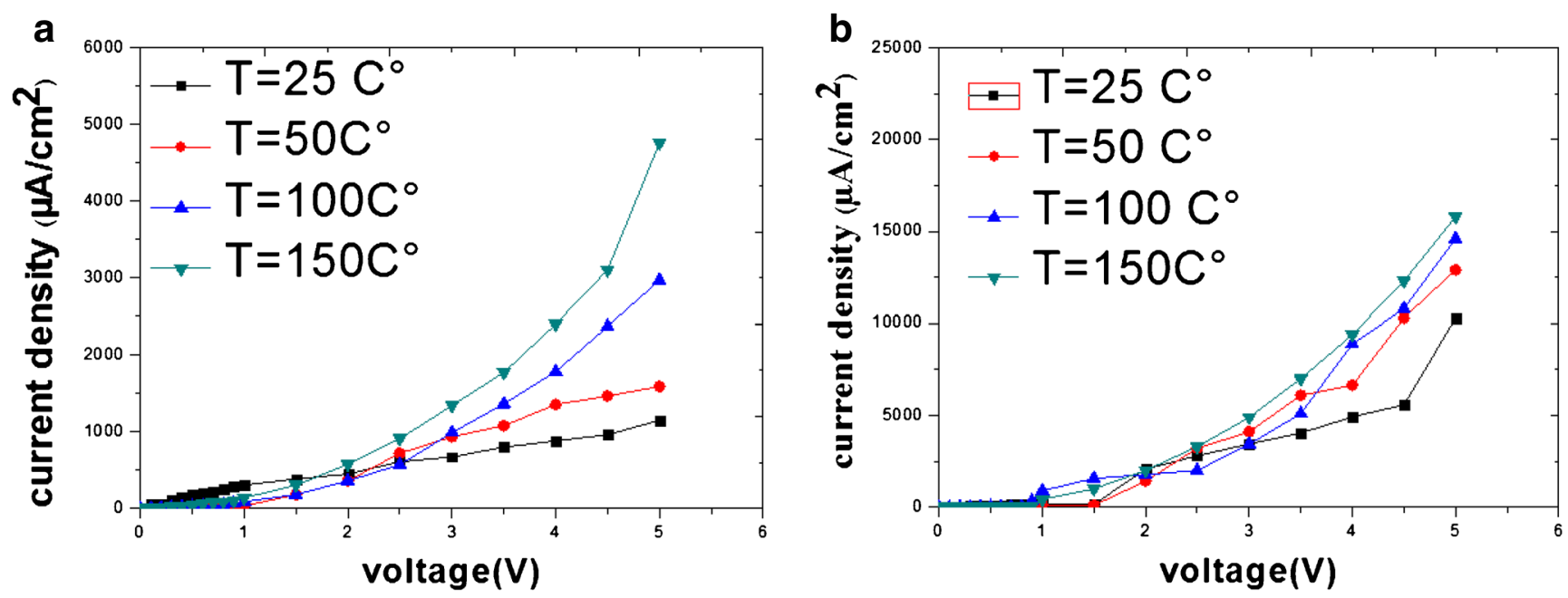

Fig. 6 illustrated the J-V characteristics for porous silicon sandwich structure (Al/nPSi/n-Si/Al) at different temperatures with presence of $5 \mathrm{ppm}$ of $\mathrm{CO} 2$ (a) sample A (b) sample B 
especially in the micro and mesoporous silicon due to the capillary condensation of the absorbate gas in the nanometer scale pores [17]. The lower thermal conductivity of the porous layer improve the condensation process within the pores inside the layer, so this modification in the dielectric constant of the porous matrix with and without condensation will lead to modifying over all electric and also the gas sensing properties.

The variation of the maximum detection sensitivity at fixed operating voltage $5 \mathrm{~V}$ with operating temperature is shown in the Fig. 7. The sensitive nature of PSi gas detection device can be defined as the change in the current density before and after exposure to the gas. The sensitivity (s) is given by Eq. (5) [18].

$S=\frac{J_{a}-J_{g}}{J_{g}} \times 100=\frac{\Delta \mathrm{J}}{J_{g}} \times 100 \%$

Where $\mathrm{J}_{\mathrm{a}}$ the essential current density of PSi sensor in the presence of atmospheric air, and $J_{g}$ is the current density after exposure to the gas.

Figure 7 of sandwich structure illustrates the maximum sensitivity for $\mathrm{CO} 2$ gas molecules, From this figures, the sensitivity of the device increased with increasing the operating temperature from 50 to $150 \mathrm{C}^{\circ}$ and the maximum

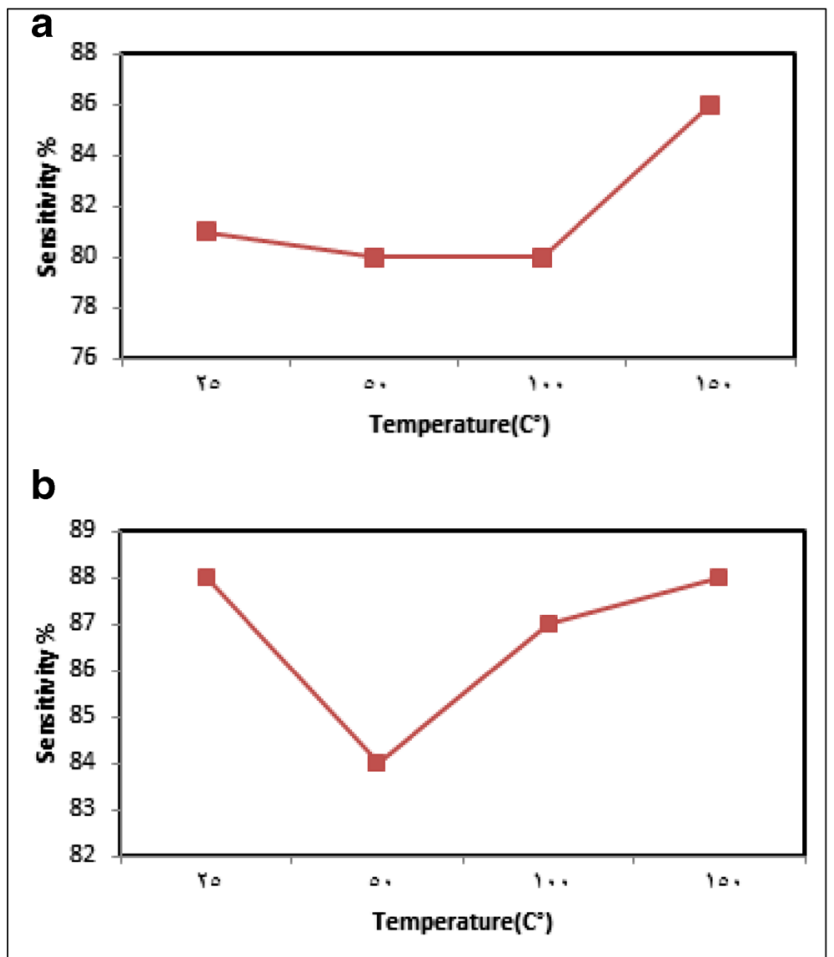

Fig. 7 shows the variation of the sensitivity at $5 \mathrm{~V}$ applied voltage for porous silicon sandwich structure $(\mathrm{Al} / \mathrm{nPSi} / \mathrm{n}-\mathrm{Si} / \mathrm{Al})$ as a function of the operating temperatures (a) sample A (b) sample B value of sensitivity was obtained at $150 \mathrm{C}^{\circ}$. By comparing Fig. 7 sample $\mathrm{A}$ and $\mathrm{B}$ it clear to us that the violet illuminated sample B has higher sensitivity than the infrared illuminated sample A.

\subsubsection{Planer Structure Configuration Mode}

The sensing mechanism of this type of the fabricated sensors was based on the recording the variation of the resistance of the porous layer. The performance of this sensor varied according to the silicon channels between pores of PSi layers (resistance). The variation of the resistance of PSi as a function of the exposure time of the planer structure gas sensor for two type of porous silicon substrates infrared illuminated PSi sample A and violet illuminated PSi sample B is shown in Fig. 8.

From the dynamic response curves, it's easier to observed that a large resistance variation is recorded when the gas out, the resistance of porous layer recovers slowly and tend to retain to the initial for both samples. The resistance of porous layer shows a decrease from a higher value to lower value and the numerical value of the resistance is varied according to the type of porous layer and the operating temperature. The resistance value, before and after exposure to $\mathrm{CO}_{2}$ gas of PSi layer has been tabulated in the following table also the response, recovery time, and the sensitivity has been tabulated in the Table 1 .

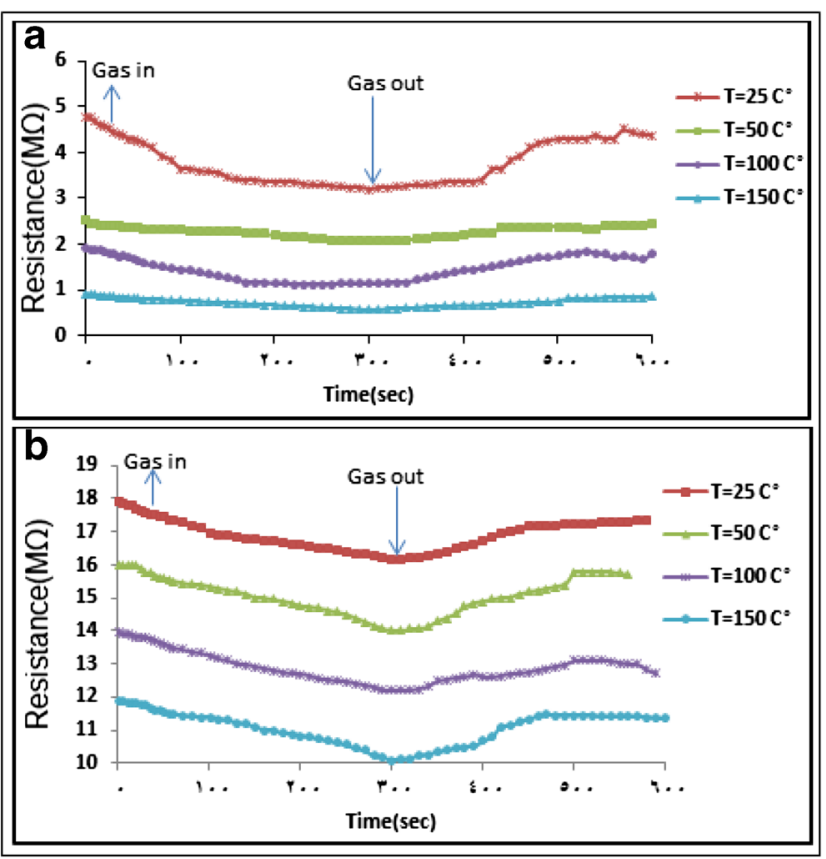

Fig. 8 Dynamic response of PSi planer structure sensor at a different temperature under $\mathrm{CO}_{2}$ concentration $(5 \mathrm{ppm})$ and (a) sample A (b) sample B 
Table 1 Shows dynamic response for porous silicon gas sensor with planer configuration

\begin{tabular}{lllllll}
\hline $\begin{array}{l}\text { Sample } \\
\text { type }\end{array}$ & $\begin{array}{l}\text { Temperature } \\
\left(\mathrm{C}^{\circ}\right)\end{array}$ & $\begin{array}{l}\text { Maximum } \\
\text { resistance }(\mathrm{M} \Omega)\end{array}$ & $\begin{array}{l}\text { Minimum } \\
\text { resistance }(\mathrm{M} \Omega)\end{array}$ & $\begin{array}{l}\text { Response } \\
\text { time(sec) }\end{array}$ & $\begin{array}{l}\text { Recover } \\
\text { time(sec) }\end{array}$ & $\begin{array}{l}\text { Maximum } \\
\text { sensitivity }\end{array}$ \\
\hline A & 25 & 4.78 & 3.2 & 125 & 170 & 33.05439 \\
& 50 & 2.52 & 2.1 & 150 & 140 & 16.66667 \\
& 100 & 1.92 & 1.113 & 160 & 200 & 42.03125 \\
& 150 & 0.915 & 0.561 & 165 & 210 & 38.68852 \\
$\mathrm{~B}$ & 25 & 17.9 & 16.17 & 240 & 170 & 9.66480 \\
& 50 & 15.99 & 14 & 200 & 200 & 12.44528 \\
& 100 & 13.94 & 12.19 & 190 & 200 & 11.47776 \\
& 150 & 11.85 & 10.1 & 195 & 110 & 13.75527 \\
\hline
\end{tabular}

The sensitivity the for planer structure PSi gas sensor is given by Eq. (6) [18]:

$\mathrm{S}=\frac{R_{a}-R_{g}}{R_{g}} \times 100=\frac{\Delta \mathrm{R}}{R_{g}} \times 100 \%$

Where $R_{a}$ is the resistance of the sensor in the presence of atmospheric air, while $R_{g}$ the device resistance after exposure to the gas.

From the Fig. 9 of planer structure illustrates the maximum sensitivity for $\mathrm{CO} 2$ gas molecules, From this figure, the sensitivity of the device increased with increasing the operating temperature from 50 to $150 \mathrm{C}^{\circ}$ and the maximum value of sensitivity was obtained at $150 \mathrm{C}^{\circ}$. By comparing Fig. 9 sample A and B it clear to us that the infrared illuminated sample A has higher sensitivity than the violet illuminated sample B. Found that efficient and best detection process of the gas sensor was fabricated in the sandwich structure by comparing with the planer mode configuration at variation the operating temperatures.

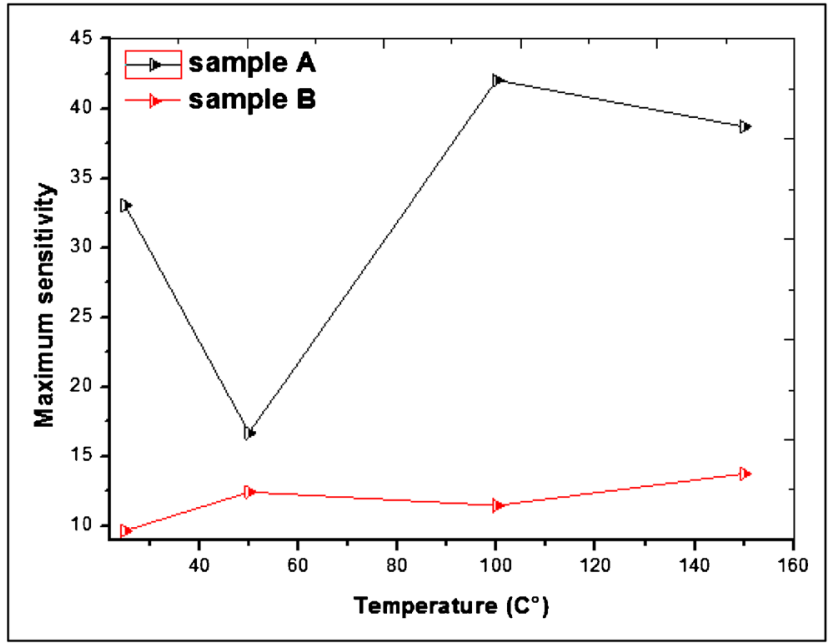

Fig. 9 shows the variation of the sensitivity at $5 \mathrm{~V}$ applied voltage for porous silicon planer structure $(\mathrm{Al} / \mathrm{nPSi} / \mathrm{n}-\mathrm{Si})$ as a function of the operating temperatures (a) sample A (b) sample B
From Table 1 the resistance of the PSi layer which lies between the deposited metallic electrode in the case of absence of $\mathrm{CO}_{2}$ gas molecule varied according to the laser, illumination wavelength, and the operating temperature, the following facts can be deduced:

1. For fixed type of PSi sample, the resistance of the PSi was decreased with increasing the temperature, this will lead to decrease the effective charge carriers, which contribute to conductance process in PSi layer.

2. The resistance of sample A PSi gas sensor samples, in general, was less than that of sample $\mathrm{B}$ because the density of the dangling bonds $\mathrm{Si}-\mathrm{H}_{\mathrm{x}}$ and the associated surface state $\mathrm{Si}-\mathrm{O}-\mathrm{Si}$ in sample A is less than that of sample B.

The performance of PSi gas sensor in the presence of gas $\mathrm{CO}_{2}$ molecule as a function of the time was varied according to the type of PSi layer. The following facts can be deduced.

1. The sensitivity of IR illuminated PSi sample A was higher than that o of the violet illumination PSi gas sensor sample B.

2. The response time PSi gas sensor was slightly varied with increasing the operating temperature from $25 \mathrm{C}^{\circ}$ to $150 \mathrm{C}^{\circ}$ ).

3. The recovery time was often higher than the response time for both PSi samples, including a fast rate for adsorption than for desorption of $\mathrm{CO}_{2}$ gas from the porous matrix.

To interpret this behavior of this sensor quantitatively, we discussed the performance of the sensor according to the activity of the dangling bonds $(\mathrm{Si}-\mathrm{H})$ associated with porous silicon. According to [19]. The conductivity and hence the resistivity was governed by the width of the channel resulting from the partial depletion of silicon located between pores. This partial depletion region was arising from the charges trapped in the surface states 
associated with the partial oxidation of porous silicon (Si$\mathrm{SiO}_{2}$ ) where $\mathrm{SiO}_{2}$ was the native silicon oxide. Due to the partial oxidation of the PSi was occurred spontaneously during the aging process. Where was a thin layer of $\mathrm{SiO}_{2}$ and associated interface states having a density $(\delta) \mathrm{cm}^{-2}$, there was a depleted region in the silicon material over the area (w). So the effective silicon channel has a width (d-2w) in which the carriers can move with applied voltage. The effect of the gas was to passivity the active dangling bonds through screening mechanism, so that $(w)$ decreased and the silicon channel increased.

\section{Conclusion}

In conclusion, we presented a simple approach to fabricated two types of porous silicon gas sensors according to a morphological parameter of the porous layer. The performance of the porous silicon gas sensor for configurations, planer, and sandwich strongly depends on the porosity and layer thickness and the silicon channel within the porous layer. For sandwich structure porous silicon gas sensor mode, the porous layer of higher porosity and lower layer thickness give us a better gas sensing process (violet illuminated sample), while planer porous silicon gas sensor for sensing process is attributed to the silicon channel rather than a porous parameter.

Open Access This article is distributed under the terms of the Creative Commons Attribution 4.0 International License (http:// creativecommons.org/licenses/by/4.0/), which permits unrestricted use, distribution, and reproduction in any medium, provided you give appropriate credit to the original author(s) and the source, provide a link to the Creative Commons license, and indicate if changes were made.

\section{References}

1. Aroutiounian VM (2013) Porous silicon gas sensors. In: Jaaniso R, Tan OK (eds) Semiconductor gas sensors. Wood head Publ. Ltd., Cambridge, pp 408-430

2. Saha H (2008) Porous silicon sensors-elusive and erudite. Int $\mathbf{J}$ Smart Sensing Intell Syst 1:34-56
3. Miller BL, Fauchet PM, Horner SR, Chan S (2012) Identification of gram negative bacteria using nanoscale silicon microcavities. J Am Chem Soc 123(47):11797-11798

4. Jeyakumaran N, Natarajan B, Ramamurthy S, Vasu V (2007) Structural and optical properties of n-type porous silicon-effect of etching time. IJNN 3(1):45-51

5. Soni RK, Bassam GR, Abbi SC (2003) Laser-controlled photoluminescence characteristics of silicon nanocrystallites produced by laser-induced etching. Appl Surf Sci 214-160

6. Canham LT (1990) Silicon quantum wire array fabrication by electrochemical and chemical dissolution of wafers. Appl Phys Lett 57:1046-1048

7. Licznerski B (2004) Thick-film gas microsensors based on tin dioxide. Bull Polish Acad Sci Tech Sci 52(1):37-42

8. Kaneyasu K, Otsuka K, Setoguchi Y, Sonoda S, Nakahara T, Aso I, Nakagaichi N (2000) A carbon dioxide gas sensor based on solid electrolyte for air quality control. Sens Actuators B 66:56-58

9. Frank J, Meixner H (2001) Sensor system for indoor air monitoring using semiconduction metal oxide and IR-absorption. Sens Actuators B 78:298-302

10. Herrán J, Mandayo GG, Castaño EJ (2008) Solid state gas sensor for fast carbon dioxide detection. Sens Actuators B 129:705709

11. Kayahan E, Bahsi ZB, Oral AY, Sezer M (2014) Porous silicon based sensor for organic vapors. In: Proceedings of the 4th international congress APMAS. Fethiye

12. Miranda CRB, Baldan MR, Beloto AF (2008) Morphological and optical characteristics of porous silicon produced by anodization process in HF-acetonitrile and HF-ethanol solutions. J Braz Chem Soc 19(4):769-774

13. Gholami M, Bhar M, Azim-Araghi ME (1986) The preparation of $\mathrm{TiO} 2$ nanoparticles and investigation of its electrical properties as $\mathrm{CO}_{2}$ gas sensor at room temperature. Elixir Chem Phys Letter 48: 9626-9628

14. Alwan AM, Abdulzahra NZ, Ahmed NM, Halim NHA (2009) Influence of rapid thermal oxidation process on the optoelectronic characteristics of PSI devices. Int J Nanoelect Mater 2:157-161

15. Alwan AM, Jawad MSM (2013) The photolumine-science characteristics of partially and fully $(\mathrm{P}-\mathrm{N})$ porous silicon. Eng Tech $\mathrm{J}$ 31(3):391-399

16. Peng C, Hirschmanand KD, Fauchet PM (1996) Carrier transport in porous silicon light-emitting devices. J Appl Phys 80:295-300

17. Dian J, Chvojka T, Vrkoslav V, Jelínek I (2008) Photoluminescence quenching of porous silicon in gas and liquid phases - the role of dielectric quenching and capillary condensation effects. Phys Stat Sol (C) 2(9):3481-3485

18. Choi Y-J, Hwang I-S, Park J-G, Choi K-J, Park J-H, Lee J-H (2008) Novel fabrication of a SnO2 nanowire gas sensor with high gas response. Nanotechnology 19:095508

19. Stievenard D, Deresmes D (1995) Are electrical properties of an aluminium-porous silicon junction governed by Dangling bonds. Appl Phys Lett 67:1570 